\title{
EXTRAHEPATIC BLOOD SUPPLY TO HEPATOCELLULAR CARCINOMA - A PICTORIAL ESSAY
}

\author{
Ton Nu Hong Hanh HUYEN ${ }^{1}$, Anh Dung HOANG ${ }^{2}$, Trong Khoan LE$^{2}$, Duc Hung NGUYEN ${ }^{3}$, \\ Hung PHUNG ${ }^{4}$, Ha Chau TRINH ${ }^{5}$, Trong Binh LE ${ }^{2 凶}$ \\ ${ }^{1}$ Department of Radiology, Hue University of Medicine and Pharmacy Hospital, Hue, Vietnam \\ ${ }^{2}$ Department of Radiology, Hue University of Medicine and Pharmacy, Hue University, Vietnam \\ ${ }^{3}$ Department of Radiology, Vietnam-Cuba Dong Hoi Friendship Hospital, Quang Binh, Vietnam \\ ${ }^{4}$ Department of Radiology, Quang Tri General Hospital, Quang Tri, Vietnam \\ ${ }^{5}$ Radiology Center, Bach Mai Hospital, Hanoi, Vietnam
}

\section{Abstract}

Transarterial chemoembolization is a standard locoregional therapy for intermediate stage hepatocellular carcinoma. The tumour is typically supplied by the hepatic arteries, however, extrahepatic collateral arteries may significantly contribute parasitic blood supply to the tumour. Large and exophytic tumour, subcapsular location, direct invasion and adhesion to adjacent organs, hepatic artery dissection, stenosis and occlusion resulting from multiple embolizations are risk factors of extrahepatic collateral arteries. Understanding the prevalence and prediction of these parasitic supplies on pre-procedural imaging is of utmost importance for optimizing technical and clinical outcomes.

Keywords: transarterial chemoembolization, parasitic supply, extrahepatic collateral.

\section{Abbreviations}

HCC - hepatocellular carcinoma

TACE - transarterial chemoembolization

\section{Résumé}

La vascularisation extra-hépatique du carcinome hépatocellulaire - un essai illustré

La chimio-embolisation trans-artérielle conventionnelle est le traitement standard et locorégional du carcinome hépatocellulaire dans les stades intermédiaires. La vascularisation du carcinome hépatocellulaire est presque exclusivement artérielle, l'artère hépatique; mais des artères à destinée initialement extra-hépatiques peuvent participer à la vascularisation de certains carcinomes hépatocellulaires. Les facteurs de risque des artères collatérales extra-hépatiques sont une large tumeur exophytique; une location sous-capsulaire; un envahissement et une adhésion aux organes adjacents; l'artère hépatique disséquante, rétrécie, obstruée par de multiples embolisations précédentes. L'objectif de cet article est de décrire les différentes artères nourrissant la tumeur. Ces artères collatérales doivent être recherchées; comprendre la prévalence et la prédiction de ces artères collatérales 
EHC - extrahepatic collateral

\section{INTRODUCTION}

Hepatocellular carcinoma (HCC) is the sixth most frequent malignancy and the third leading cause of cancer mortality, contributing to $11 \%$ of cancer-related death worldwide ${ }^{1-3}$. According to the Barcelona Clinic Liver Cancer staging system, trans-arterial chemoembolization (TACE) is the standard local-regional therapy for a large group of HCC patients of the intermediate stage who are not candidates for $\mathrm{cu}$ rative treatment ${ }^{2-6}$. Moreover, TACE can be indicated in patients with early-stage $\mathrm{HCC}$, in whom the recommended treatments are not feasible or have failed and used as a bridging therapy or downstaging for pending liver transplant or resection ${ }^{2,3}$. TACE is based on the principle of intra-arterial injection of an antineoplastic agent, followed by occlusion of the tumour feeding arteries. The aim of TACE is to produce tumour ischemia and concentrate the antineoplastic agent in the tumour, reducing its systemic toxicity ${ }^{3,7,8}$.

Familiarity with variations in the hepatic arterial anatomy is of the utmost importance when performing TACE. Classically, HCC is mainly vascularized by the hepatic arteries (HA), however, numerous extrahepatic collateral (EHC) arteries may also provide parasitic blood supply to the tumour in certain circumstances and even when the HA is patent ${ }^{9,10}$. Generally, HCCs in contact with the liver capsule and large size have an overall likelihood of being supplied by EHC arteries ${ }^{3,11}$. The estimated prevalence of EHC arteries supplying HCC ranges between 17\% and $30.8 \%{ }^{4,12}$, and $\mathrm{EHC}$ development can be responsible for local tumour recurrence ${ }^{13,14}$. Moreover, EHC arteries can be the source of bleeding in the spontaneous rupture of an $\mathrm{HCC}^{15}$. TACE of HCC through $\mathrm{HA}$ and $\mathrm{EHC}$ arteries is therefore essential for achieving optimal therapeutic outcomes ${ }^{4}$. Embolization of these EHC arteries is generally safe though complications of the skin, diaphragm, gallbladder, and other organs have been reported ${ }^{4,7,9,11,13,14,16}$. However, determining the presence and catheterizing $\mathrm{EHC}$ arteries is sometimes challenging. Thus, every interventional radiologist who performs TACE should be aware of the wide spectrum of EHC arteries, their anatomy, prevalence, and potential communication. In this article, we review the likelihood of EHC development with angiographic illustrations. sur les examens d'imagerie du bilan pré-thérapeutique est de la plus grande importance pour l'optimisation de la technique et du résultat.

Mots-clés: chimio-embolisation trans-artérielle, artères collatérales extra-hépatiques.

\section{Prevalence of EHC}

Various EHC arteries can potentially supply an HCC. Nonetheless, the presence of parasitic blood supply to HCC can be predicted based on tumour location, tumour characteristics (exophytic growth, extra capsular invasion), hypertrophy of a potential EHC artery, a peripheral defect in the tumour blush that smaller than expected tumour size at pre-procedural imaging, and marked attenuation of the HA on conventional angiography ${ }^{4,9}$. The prevalence of EHC arteries varies among studies, in which the inferior phrenic artery (IPA) and the omental artery (OMA) remain the most common ${ }^{4,8}$. Moustafa et al reported the frequency of EHC arteries as follows: right IPA (RIPA 70-83\%), left inferior phrenic artery (LIPA 12\%), OMA $13 \%$, right internal mammary artery (RIMA 8\%), left internal mammary artery (LIMA $1 \%$ ), right suprarenal artery (RSRA 9-11\%), right renal artery (RRA 12\%), intercostal artery (ICA 6-8\%), cystic artery (4\%), right and left gastric artery (RGA, LGA 3-4\%), lumbar artery (2\%), superior mesentery artery (SMA 1.5\%) $)^{3,4,9,14}$.

EHC arteries can be divided into 2 groups based on their origins: the upper abdomen group (IPA, OMA, RSRA, renal artery, cystic artery, gastric arteries, SMA, gastroduodenal artery and lumbar artery) and lower thorax group (IMA, ICA) ${ }^{12}$.

\section{Upper Abdominal Extrahepatic Collateral Arteries}

Inferior Phrenic Arteries (IPA)

The right IPA (Figure 1) is the most common source of EHC blood supply for HCC and frequently supplies HCCs located in the bare area of the liver ${ }^{17}$. Gwon et al found that origins of the IPA are the celiac axis $(39.7 \%)$, aorta $(38.6 \%)$, renal artery $(15.4 \%)$, left gastric artery $(3.7 \%)$, hepatic artery $(2.1 \%)$, superior mesenteric artery $(0.3 \%)$ and contralateral IPA $(3 \%)^{17}$. The RIPA can anastomose with intrahepatic arteries at the caudate lobe and the posterior segment while the LIPA can communicate with those in the lateral segment ${ }^{8}$. In addition, the IPA can communicate with other adjacent arteries, such as RIMA, LIMA, RICA, LGA and RSRA, and facilitates an EHC blood supply to hepatic lesions located in the liver segment VII or those contact with the 

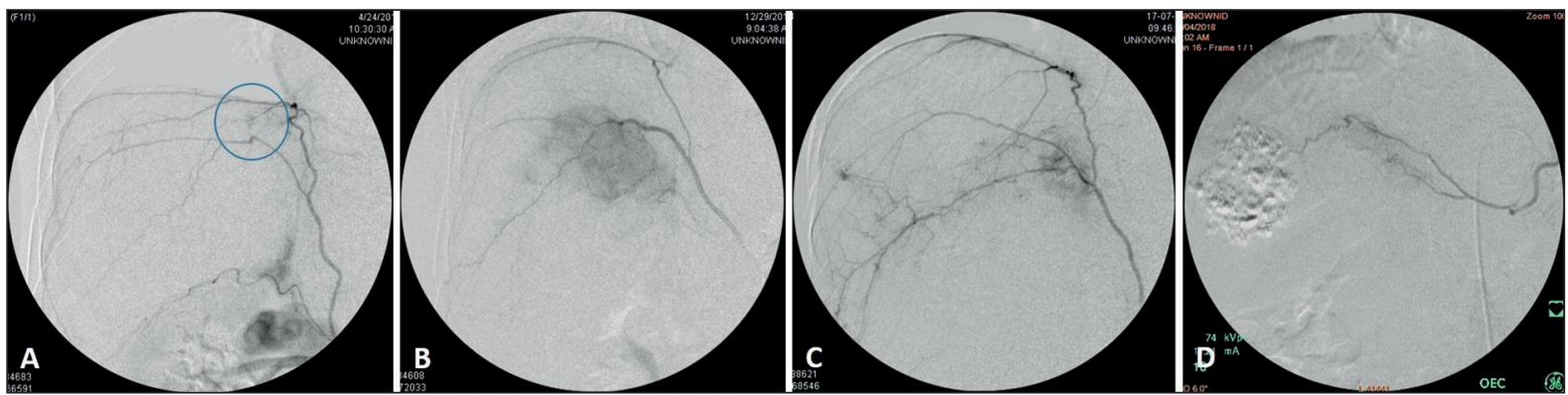

Figure 1. Extrahepatic parasitic supply from the RIPA. (A): ascending (anterior) branch, (B): descending (posterior) branch, (C): multiple tumour staining, (D): superior adrenal branch
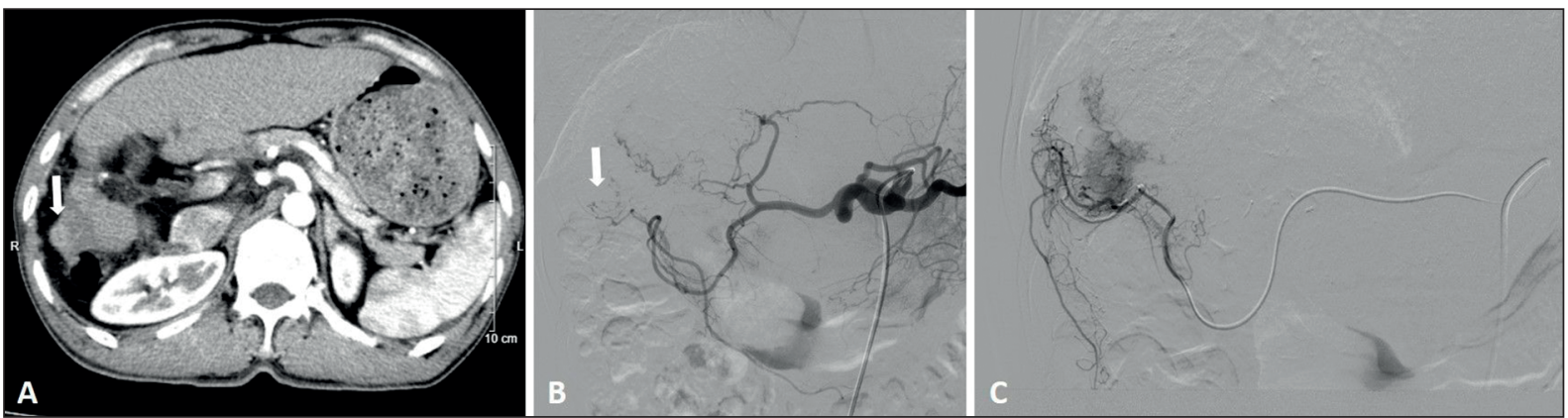

Figure 2. A 55-year-old male received multiple sessions of TACE due to HCC. (A) Follow-up CT scan showed a hypervascular nodule at the inferior margin of the posterior segment (arrow). (B) The nodule was supplied by omental branches from the gastroepiploic artery. (C) Superselective omental angiography clearly demonstrated a tumour staining.

right hemidiaphragm ${ }^{4,13}$. It is the main $\mathrm{EHC}$ artery which may contribute up to $50 \%$ of EHC arteries ${ }^{3}$. Notably, attenuation of the IPA can result in the augmentation or exaggeration of another adjacent EHC supply to $\mathrm{HCC}^{13}$. The RIPA is highly suspected when (i) the lesion locates in the liver dome or caudate lobe (88\% of EHC arterial blood supply) and (ii) there is an asymmetry between the RIPA and LIPA seen on pre-procedural CT due to hypertrophied RIPA. The LIPA should be taken into consideration when the lesion abuts the left hemidiaphragm or locates in segments II and III of the left hepatic lobe ${ }^{4,17}$. IPA embolization is usually feasible and safe. Complications may include hiccups, shoulder pain, lung consolidation, pleural effusion, esophagitis, gastritis, and gastric ulceration ${ }^{3,4,9}$. Diaphragmatic weakness is radiologically evident in $40 \%$ of the cases, yet mostly asymptomatic ${ }^{8}$.

\section{Omental artery (OMA)}

This is the second most common EHC artery (Figure 2) which can provide parasitic supply to hepatic lesions located at the segment IV ${ }^{4,8}$. However, due to the high mobility of the omentum, this EHC artery can supply HCC of any segment ${ }^{3,8}$. Multiple omental branches usually originate from the gastroepiploic artery or the dorsal pancreatic artery ${ }^{8}$. The OMA is usually hypertrophied to be detectable on CT when supplying an $\mathrm{HCC}^{8}$. The embolization of the OMA is generally safe with several reported complications such as omental infarction and fat necrosis ${ }^{3,4,8}$.

\section{Cystic Artery}

The cystic artery, a branch of the right HA, can provide parasitic feeders to HCC located adjacent to the gallbladder fossa ${ }^{4,9}$. It can also provide an EHC arterial supply to HCC located in other portions of the right hepatic lobe and in the medial segment of the left hepatic lobe (Figure 3). This occurs more frequently when the HA is attenuated owing to prior TACE procedures. Selective catheterization is strongly recommended to avoid non-target embolization. Ischemic cholecystitis and gallbladder infarction are reported complications ${ }^{3,4}$.

\section{Suprarenal arteries}

Suprarenal arteries consist of 3 sources (superior, middle, and inferior) that supply the adrenal gland ${ }^{8}$. The right suprarenal arteries may provide parasitic supply to HCC which grows inferomedial and contacts the adrenal gland ${ }^{8}$ (Figure 4). TACE through the suprarenal arteries is generally safe, given the rich blood supply to the adrenal gland, though adrenal insufficiency and infarction have been encountered ${ }^{4,8}$. 

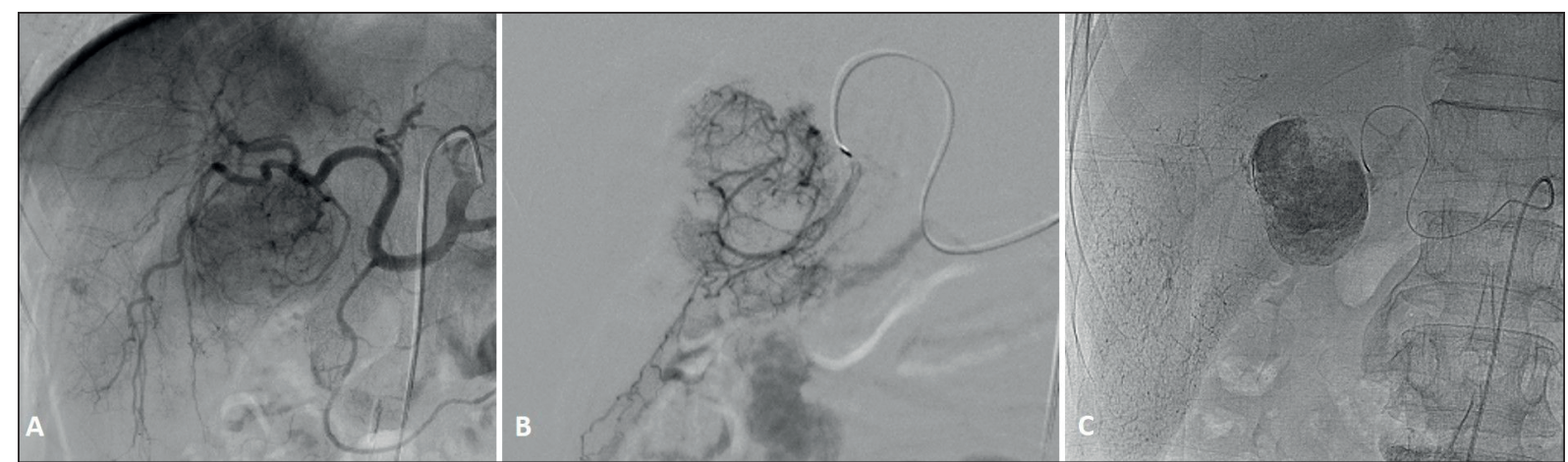

Figure 3. Extrahepatic parasitic supply from the cystic artery. (A) Celiac angiogram showed a tumour staining at the segment V, partially supplied from the cystic artery. (B) Super selective cystic arteriogram showed tumour network. (C) Dense Lipiodol uptake after chemoembolization.
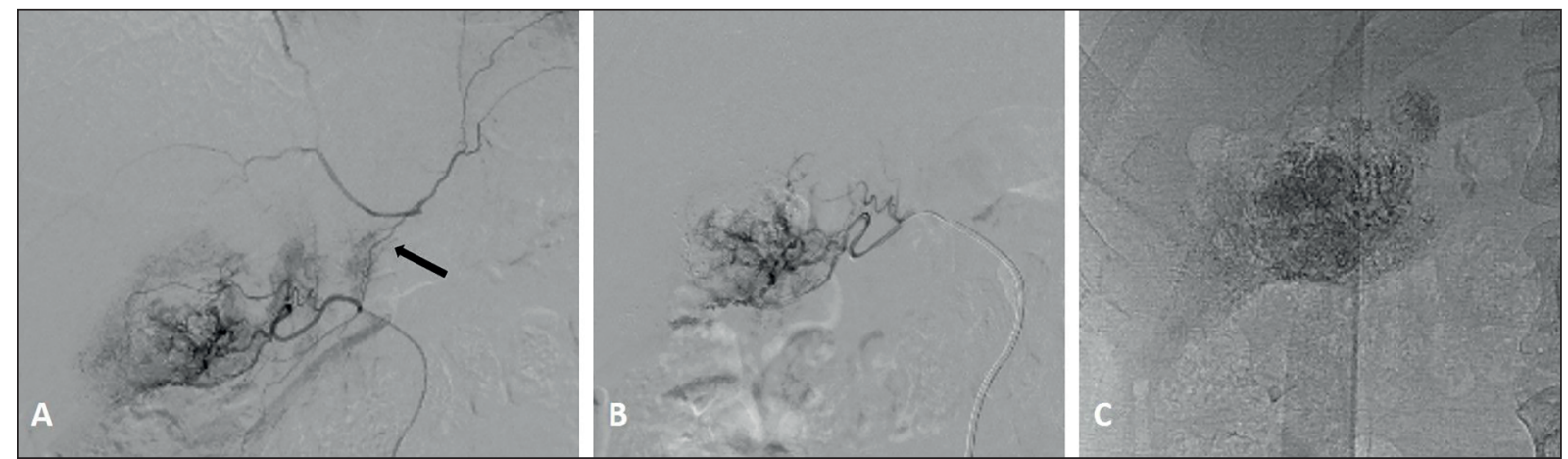

Figure 4. Parasitic supply from the right suprarenal artery. (A) Tumour staining was evident on suprarenal arteriogram. Note a connection with bilateral inferior phrenic artery (arrow).

(B) Super selection of the right suprarenal artery before embolization. (C) Lipiodol uptake.
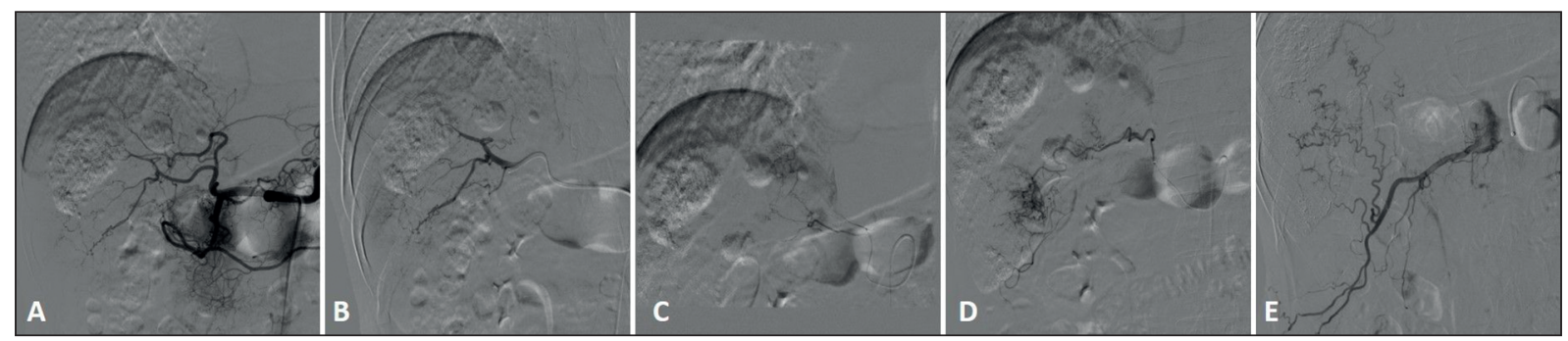

Figure 5. Multiple hepatic and extrahepatic parasitic supplies to a multifocal HCC. (A) Celiac angiogram of the $4^{\text {th }}$ TACE showed tumour staining at the posterior segment and multiple Lipiodol uptake from previous TACE. (B) Selective embolization of the posterior segment branch. (C): Tumour staining from the adrenal artery injection.

(D) Selective embolization of the right renal capsular artery. (E) Multiple tumour staining from the lumbar artery.
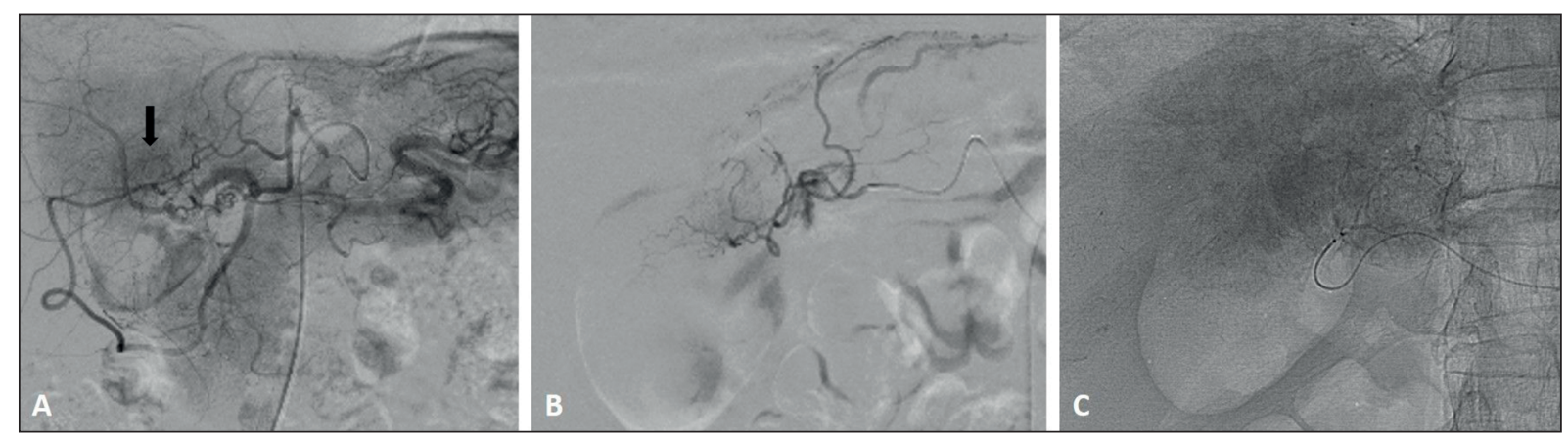

Figure 6. Marginal recurrence of an HCC after left lobectomy. (A) Celiac angiogram demonstrated a tumour staining at the segment $\mathrm{V}$ (arrow), supplied by the right hepatic artery and the left gastric artery.

(B) Super selective chemoembolization of the left gastric artery. (C) Lipiodol uptake. 


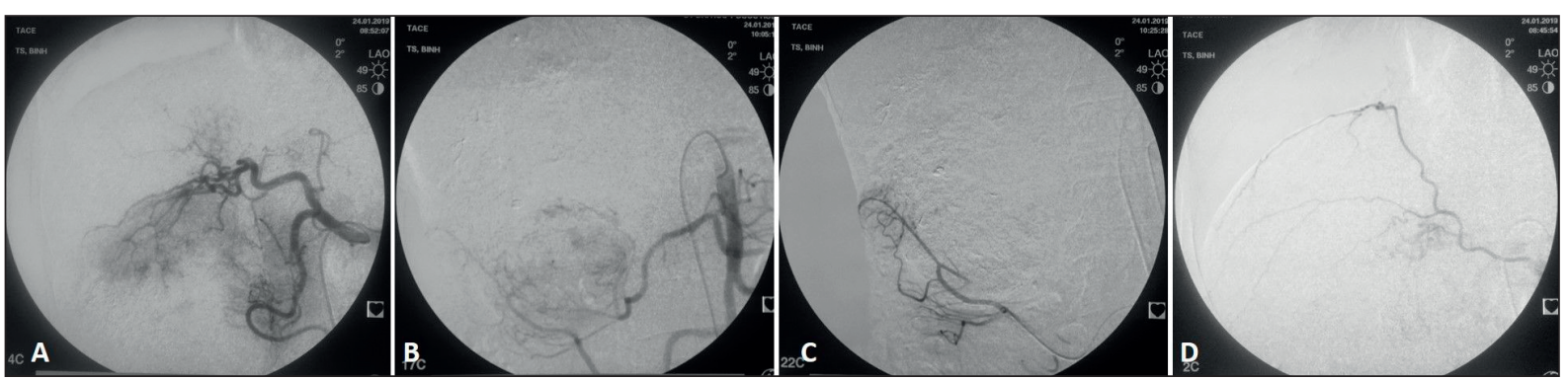

Figure 7. A 57-year-old man with a huge exophytic HCC. Common hepatic arteriogram shows tumour staining at the posterior segment (A). Parasitic supplies from the right colic branch (B) and the ileocolic branch (C) of the superior mesenteric artery, and from the superior adrenal branch of the right inferior phrenic artery (D).

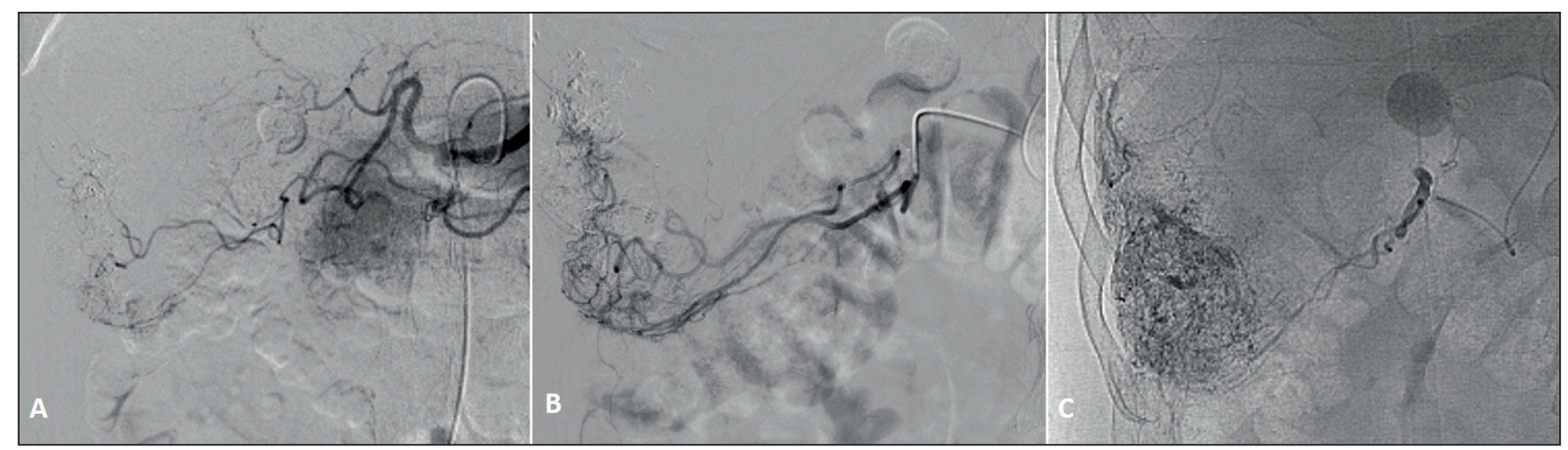

Figure 8. Repeat TACE for a residual HCC supplied by the gastroduodenal artery (GDA).

(B) Super selective embolization of the GDA. (C) Full Lipiodol uptake on spot image.
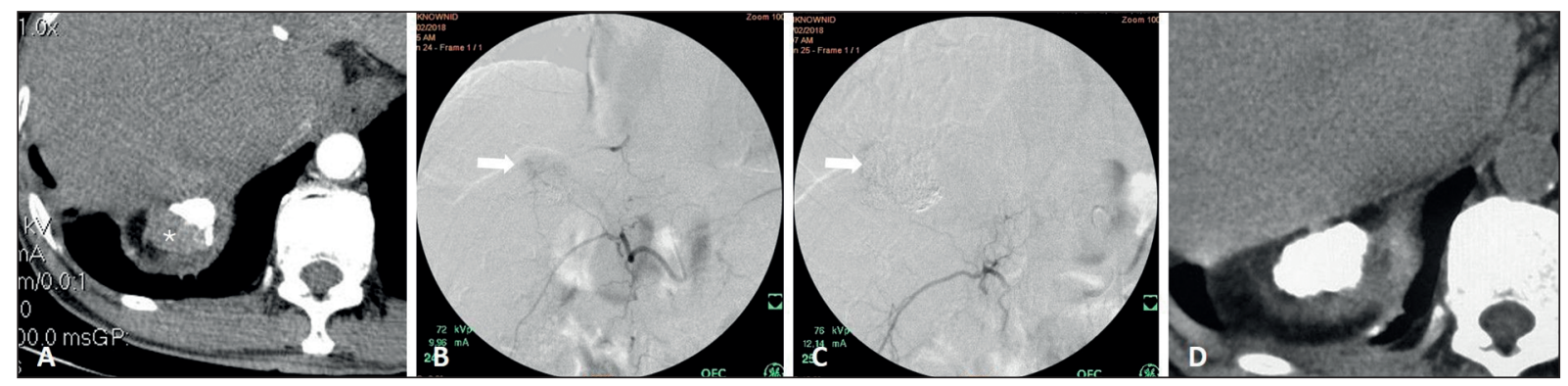

Figure 9. A 56-year-old with multifocal HCC undergoing multisession TACE. (A) Follow-up CT scan shows incomplete Lipiodol uptake and a viable exophytic tumour (asterisk) at the dome area after previous embolization of the RIPA. (B) Selective angiogram of the right lumbar artery reveals a tumour staining (arrow).

(C,D) Dense Lipiodol uptake on final angiogram and follow-up CT.

\section{Right renal artery}

The right renal arteries may provide parasitic blood supply to HCC at segments VI and VIII [8]. The superior capsular artery is also reported to have an important role in providing these feeders (Figure 5). Detection of an EHC arterial supply from the right renal artery on pre-TACE CT scans is limited and accounts for about $5 \%$ of cases of EHC arteries supplying HCC. The embolization of these feeders has a wide range of technical success and is generally safe without documented complications ${ }^{4}$.

\section{Gastric Arteries}

The right and left gastric arteries may provide blood flow to hepatic lesions in close contact with the lesser curvature of the stomach (segment I, II, III). Again, super-selective embolization is necessary to avoid gastric ischemia ${ }^{4,18}$ (Figure 6).

\section{Superior Mesenteric Artery (SMA)}

Exophytic HCC at the inferior tip of the liver (segments V and VI) or in close contact with adjacent bowel loop may receive an EHC arterial supply from the right colic branch or even the ileocolic branch of the $\mathrm{SMA}^{8}$ (Figure 7). Detection of parasitic feeders from the terminal branches of the SMA with CT is limited, therefore, tumour location is usually the clue for prediction ${ }^{4}$. Distal embolization is recommended to avoid bowel ischemia ${ }^{4}$. 

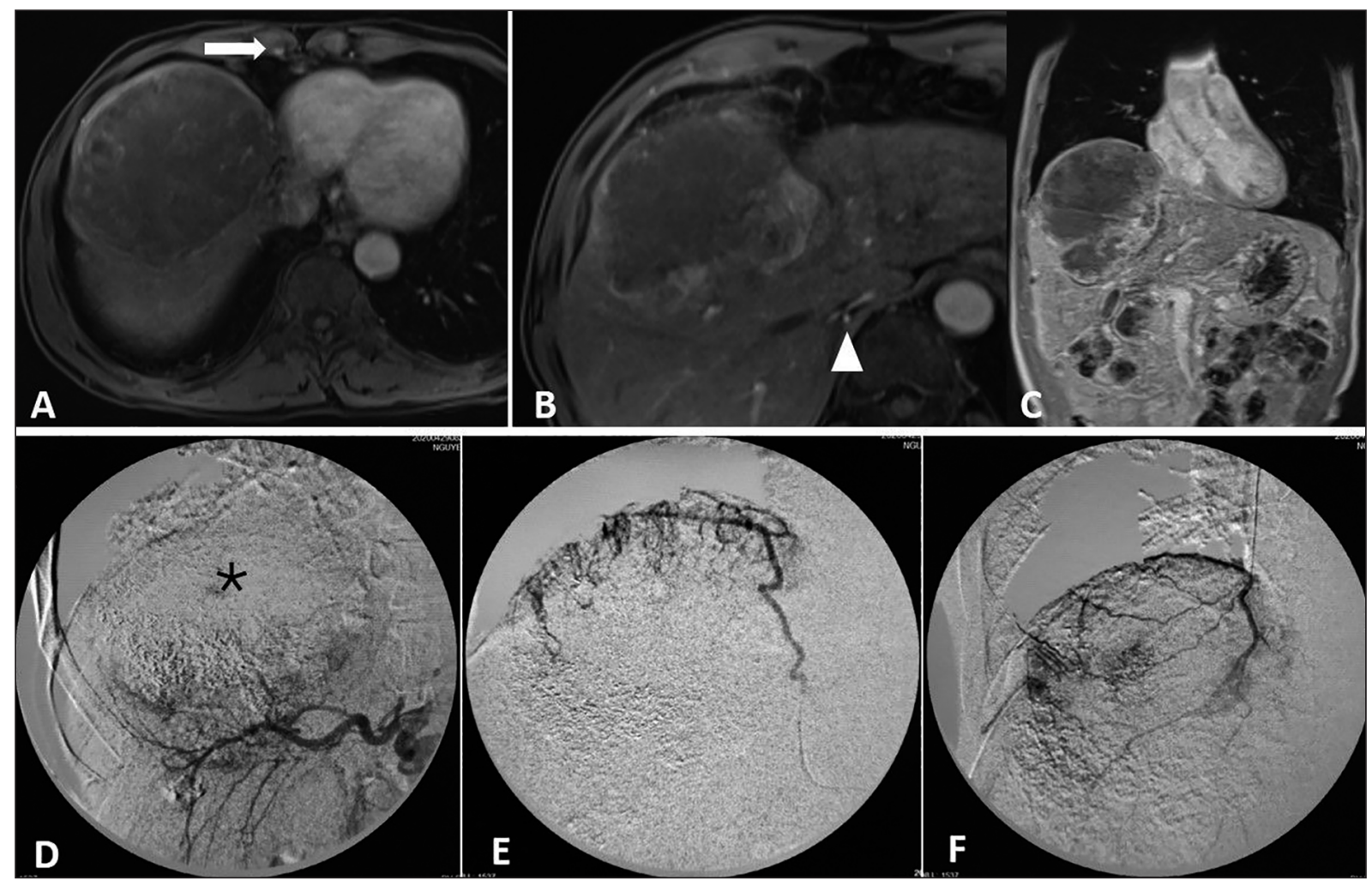

Figure 10. Embolization of a huge exophytic HCC abutting the right hemidiaphragm. Follow up magnetic resonance images after the $1^{\text {st }}$ TACE demonstrated arterial enhancement of viable tumours (A, B) and washout (C).

Note the hypertrophy of the right IMA (arrow) and RIPA (arrowhead) compared to their contralateral counterparts.

(D) Celiac angiogram showed multifocal tumour staining corresponding to viable tumours. Incomplete Lipiodol uptake (asterisk) was also noted. (E) Super selective RIPA angiogram showed massive tumour staining.

(F) Catheterization and embolization of the right IMA which also contributed blood supply to the tumour.

\section{Gastroduodenal Artery (GDA)}

HCC at the medial aspect of the segments V and VI or in close contact with the duodenum may receive an EHC arterial supply from the gastroduodenal artery or its branches $^{8}$ (Figure 8 ). Post hepatectomy is another favorable factor of EHC development due to neovascularization and stimulation of vascular endothelial growth factor ${ }^{3}$. Acute pancreatitis, upper gastrointestinal inflammation, ulceration, and even perforations are possible complications of non-targeted embolization of GDA branches ${ }^{4,8}$.

\section{Lumbar Arteries}

The lumbar arteries can anastomose with the lower ICAs and may provide a parasitic arterial blood supply to HCC lesions located at segment $\mathrm{VI}^{4}$. This vascularization often occurs at a late stage after multiple sessions of embolization (Figure 9) ${ }^{3}$. The technical success of TACE of lumbar arteries supplying HCC is limited (40\%) owing to the various anastomoses between the lumbar and spinal arteries. Identifying this communication on angiography is crucial to decide if TACE is indicated ${ }^{4}$.

\section{Lower Thoracic Extrahepatic Collateral Arteries} Internal mammary artery (IMA)

The right IMA accounts for $8 \%$ of EHC arterial supply to an $\mathrm{HCC}^{4}$ (Figure 10). Small branches from the superior epigastric artery, one of a terminal branch of the IMA, extend into the falciform ligament and communicate with the $\mathrm{HA}^{8}$. Parasitic supply from IMA is suspected when the vessel is hypertrophied and there is a large HCC abutting the ventral aspect of the diaphragm or locating in the segment II, III, IV, and VIII ${ }^{3,8}$. Though complications of IMA embolization are usually mild and self-limiting, TACE should be performed super-selectively to avoid any non-targeted embolization of the anterior chest wall cutaneous branches. Chest wall pain, skin rash, pleural effusion, basal atelectasis, and even skin necrosis have been reported ${ }^{4,8}$.

\section{Intercostal Arteries (ICA)}

Generally, large HCCs in the subcapsular region of the segment VI and VII and in contact with the anterior abdominal wall are prone to recruit a blood supply from an ICA, though this is not a common parasitic supply (Figure 11) $3,4,8,19$. Up to $83 \%$ 


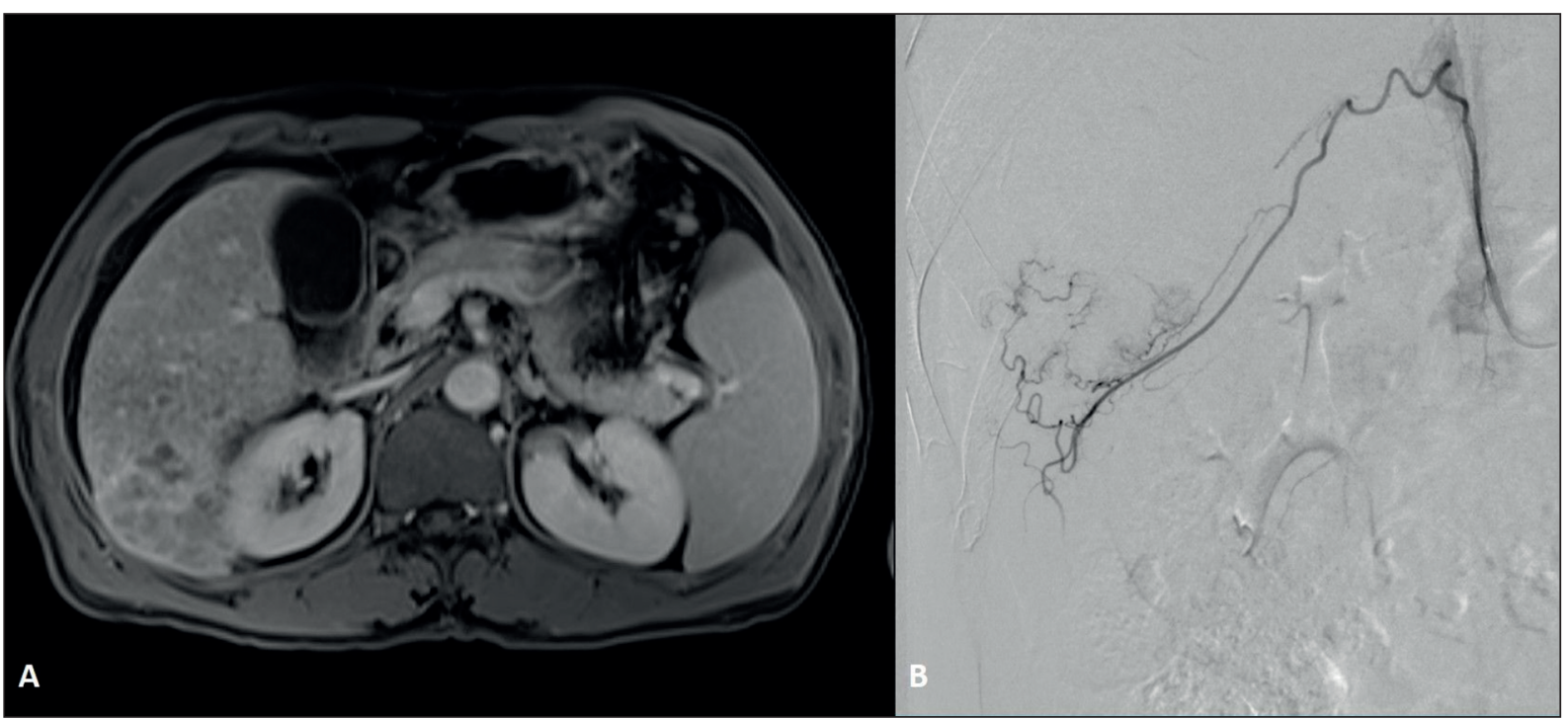

Figure 11. Parasitic supply from the right subcostal artery. (A) Axial MR image showed a hyper vascular mass locating at the posterior segment, invading the abdominal wall.

(B) Super selective right subcostal arteriogram confirmed parasitic supply to the tumour.

of HCCs supplied by an ICA are found in patients who have a history of TACE through the IPA. This could be explained by the anastomosis between the lower posterior ICAs and the IPA at the diaphragmatic insertion ${ }^{4}$. The right $8^{\text {th }}-11^{\text {th }}$ ICAs are the most common sources of EHC supplies to hepatic tumours $^{3}$. Suggestive CT findings were large HCC $(10 \mathrm{~cm})$, subcapsular location, defect in Lipiodol retention, HCC abutting abdominal wall, and hypertrophy ${ }^{19}$. Technically, a microcatheter should be advanced further to the ascending vertical limb of the ICA before embolization to prevent regurgitation, otherwise, TACE is contraindicated due to the risk of spinal cord ischemia ${ }^{4,8}$. Complications may include shoulder pain, pruritus, erythema, and skin necrosis ${ }^{3}$.

\section{How to PREDICt AN EHC}

Generally, the main cause of EHC development was considered HA occlusion by surgical ligation, mechanical injury or multiple TACE procedures ${ }^{7,8}$. Recent studies have reported interruption or dissection of the HA by multiple TACE procedures to be the principal cause of the formation of EHC vessels ${ }^{7,13}$. Patient age, atherosclerosis, patency of the HA, tumour size, anatomic location, direct invasion or adhesions to adjacent organs and previous treatment (surgical resection, ligation, TACE) are considered the causative factors of EHC formation ${ }^{4,7-9,13,14}$. According to previous studies, tumour size is a consistent predictor of EHC development ${ }^{7,9,11,13}$. A positive correlation between tumour location, tumour size $(>5 \mathrm{~cm})$ and number of previous TACE and presence of parasitic blood supply to HCC has been observed $^{4,7,8,12}$. Peripheral tumours and tumours at the bare area of the liver, even small in size, have been found to have a higher incidence of EHC arterial supply $y^{4,8,9}$. On the other hand, patients who have never undergone TACE or who have a patent HA may also have EHC arterial supplies ${ }^{4,7}$. Tumour hypervascularity is another important factor in the development of EHC arteries ${ }^{4}$. Another study investigated that the non-intact pseudo-capsule group had a higher incidence of ectopic blood supply than the intact pseudo-capsule group ${ }^{12}$. Kim et al suggested EHC vessels when (1) a tumour grows exophytic or invades adjacent organs, (2) a tumour was in contact with the ligaments and bare area of the liver, (3) a hypertrophied EHC vessel is seen on a computed tomographic (CT) scan, (4) a peripheral defect of iodized oil retention within a tumour is seen during chemoembolization or on a follow-up CT scan, (5) a local recurrence develops at the peripheral portion of the treated tumour during follow-up, or (6) a sustained elevation in serum alpha-fetoprotein level is noted despite adequate embolization of the $\mathrm{HA}^{10}$. Additionally, several arteries close to the liver, which are initially non-hepatic in nature, can be recruited by the tumour for its vascular supply ${ }^{3}$.

\section{Conclusion}

Parasitic supply from EHC arteries is common in clinical practice. Detection and embolization of these vessels are essential to optimize treatment outcomes. Tumour size, location, vessel hypertrophy, 
attenuated HA and multiple embolizations are suggestive of EHC pathway. Careful review of pre-procedural CT is strongly recommended before any procedure. Super-selective embolization where possible and understanding any vascular communication and anastomoses are crucial for a safe and effective TACE.

\section{Author Contributions:}

T.N.H.H.H. conceived the original draft preparation. T.B.L. and T.K.L. were responsible for conception and design of the review. T.B.L., A.D.H., D.H.N., H.P. and H.C.T. were responsible for the data acquisition. T.N.H.H.H. and T.B.L. were responsible for the collection and assembly of the articles/published data, and their inclusion and interpretation in this review. All authors contributed to the critical revision of the manuscript for valuable intellectual content. All authors have read and agreed with the final version of the manuscript.

\section{Compliance with Ethics Requirements:}

„The authors declare no conflict of interest regarding this article"

„The authors declare that all the procedures and experiments of this study respect the ethical standards in the Helsinki Declaration of 1975, as revised in 2008(5), as well as the national law. Informed consent was obtained from all the patients included in the study"

"No funding for this study"

\section{Acknowledgements:}

None

\section{References}

1. Tang A, Hallouch O, Chernyak V, Kamaya A, Sirlin CB. Epidemiology of hepatocellular carcinoma: target population for surveillance and diagnosis. Abdom Radiol (NY) 2018; 43:13-25.

2. Raoul JL, Forner A, Bolondi L, Cheung TT, Kloeckner R, de Baere T. Updated use of TACE for hepatocellular carcinoma treatment: How and when to use it based on clinical evidence. Cancer Treatment Reviews 2019; 72:28-36.

3. Cazejust J, Bessoud B, Colignon N, Garcia-Alba C, Planche $\mathrm{O}$, Menu Y. Hepatocellular carcinoma vascularization: from the most common to the lesser known arteries. Diagnostic and Interventional Imaging 2014; 95:27-36.

4. Moustafa AS, Abdel Aal AK, Ertel N, Saad N, DuBay D, Saddekni S. Chemoembolization of hepatocellular carcinoma with extrahepatic collateral blood supply: anatomic and technical considerations. Radiographics: a review publication of the Radiological Society of North America, Inc 2017; 37:963-77.

5. Marrero JA, Kulik LM, Sirlin CB, et al. Diagnosis, staging, and management of hepatocellular carcinoma: 2018 practice guidance by the American Association for the Study of Liver Diseases. Hepatology 2018; 68:723-50.

6. EASL. EASL Clinical Practice Guidelines: Management of hepatocellular carcinoma. Journal of hepatology 2018; 69:182-236.

7. Zhao Y, Fang Z, Luo J, et al. Evaluation of extrahepatic collateral arteries in hepatocellular carcinoma in three independent groups in a single center. Experimental and Therapeutic Medicine 2015; 10:2366-74.

8. Cheng LF, Ma KF, Fan WC, Yung AW, Li TM, Wong CS. Hepatocellular carcinoma with extrahepatic collateral arterial supply. Journal of Medical Imaging and Radiation Oncology 2010; 54:26-34.

9. Miyayama S, Matsui O, Taki K, et al. Extrahepatic blood supply to hepatocellular carcinoma: angiographic demonstration and transcatheter arterial chemoembolization. Cardiovascular and Interventional Radiology 2006; 29:39-48.

10. Kim HC, Chung JW, Lee W, Jae HJ, Park JH. Recognizing extrahepatic collateral vessels that supply hepatocellular carcinoma to avoid complications of transcatheter arterial chemoembolization. Radiographics: a review publication of the Radiological Society of North America, Inc 2005; 25 Suppl 1:S25-39.

11. Chung JW, Kim HC, Yoon JH, et al. Transcatheter arterial chemoembolization of hepatocellular carcinoma: prevalence and causative factors of extrahepatic collateral arteries in 479 patients. Korean Journal of Radiology 2006; 7:257-66.

12. Guang-wen Chen BS, Zhen-lin Li, Yuan Yuan. Ectopic blood supply of hepatocellular carcinoma as depicted by angiography with computed tomography: associations with morphological features and therapeutic history. PLoS One 2013; 8.

13. Li Q, Ao GK, Duan F, Wang ZJ, Yan JY, Wang MQ. Incidence and therapeutic frequency of extrahepatic collateral arteries in transcatheter arterial chemoembolization of hepatocellular carcinoma: Experience from 182 patients with survival time more than 3 years. European Journal of Radiology 2015; 84:2555-63.

14. Huang Y, Jia Z, Tu J, Shen T, Tian F, Jiang G. Supplemental conventional transarterial embolization/chemoembolization therapy via extrahepatic arteries for hepatocellular carcinoma. Journal of Cancer Research and Therapeutics 2017; 13:720-4.

15. Nishiyama T, Kamo M, Horiuchi S, et al. Extrahepatic collateral artery extravasation in patients with ruptured hepatocellular carcinoma. Journal of Vascular and Interventional Radiology: JVIR 2018; 29:564-7.

16. Lokken RP, Fidelman N, Kolli KP, Kerlan RK, Jr. Safety and efficacy of doxorubicin drug-eluting embolic chemoembolization of hepatocellular carcinoma supplied by extrahepatic collateral arteries. Journal of Vascular and Interventional Radiology: JVIR 2016; 27:1698-704.

17. Gwon DI, Ko GY, Yoon HK, et al. Inferior phrenic artery: anatomy, variations, pathologic conditions, and interventional management. Radiographics: a review publication of the Radiological Society of North America, Inc 2007; 27:687-705.

18. Park JH, Jeon UB, Kim TU, et al. Chemoembolization for hepatocellular carcinoma supplied by the right gastric artery. Gastrointestinal Intervention 2015; 4:124-6.

19. Park SI, Lee DY, Won JY, Lee JT. Extrahepatic collateral supply of hepatocellular carcinoma by the intercostal arteries. Journal of Vascular and Interventional Radiology: JVIR 2003; 14:461-8. 